\title{
Sozialpädagogik/Sozialarbeit in Deutschland Geschichtsschreibung und Armenpflege ${ }^{1}$
}

\author{
Social Pedagogy/Social Work in Germany
}

\begin{abstract}
The history of social pedagogy and social work (for more than 35 years embraced by the common name of "societal work") is an aspect of the development of the German society. Their existence is determined by the structural durability of social inequality. The submitted text reconstructs the historical and systemic conditions for the development of social pedagogy/societal work across the German lands. The analysis focuses around the thesis that social pedagogy practiced in bourgeois-capitalist social formation, accepting the capitalist model of socialization, has to have a special way of dealing with the evolution of relations between the society/social structures and the individual, and thus the conditions of the constitution of subjectivity, the ability to operate, etc. The historical development shows that social pedagogy/social work has been exposed to political instrumentalization.
\end{abstract}

Keywords: history of social pedagogy and societal work, capitalist society, possibility of emancipation, political productivity.

\section{Pedagogika społeczna/praca socjalna w Niemczech}

\section{Abstrakt}

Historia pedagogiki społecznej i pracy socjalnej (od ponad 35 lat obejmowanych wspólną nazwą „praca społeczna”) stanowi aspekt rozwoju niemieckiego społeczeństwa. Ich istnienie warunkuje strukturalna trwałość nierówności społecznych. Przed-

\footnotetext{
* Otto-Friedrich Univeristät Bamberg.

** Bergische Universität Wuppertal.

1 Erweiterte und geänderte Fassung von Veröffentlichung in Englisch, Spanisch: Trabajo Social En Alemania, Social Pedagogy/Social Work in Germany, in: Pedagogia Social. Revista Interuniversitaria, 27 (2016), 19-44. Die NOWIS-Redaktion möchte die Autoren für die Bereitstellung des Textes zur Veroffentlichung danken.
} 
łożony tekst rekonstruuje historyczne i systemowe warunki rozwoju pedagogiki społecznej/pracy socjalnej na ziemiach niemieckich. Analiza koncentruje się wokół tezy, iż pedagogika społeczna uprawiana w mieszczańsko-kapitalistycznej formacji społecznej, przyjmującej kapitalistyczny wzór uspołecznienia, musi w szczególny sposób zajmować się kształtowaniem stosunków między społeczeństwem/strukturami społecznymi a jednostką, a tym samym warunkami konstytucji podmiotowości, zdolnością do działania itd. Rozwój historyczny pokazuje, że pedagogika społeczna/praca socjalna narażona była (i nadal jest) na polityczną instrumentalizację.

Słowa kluczowe: historia pedagogiki społecznej i pracy socjalnej, społeczeństwo kapitalistyczne, możliwości emancypacji, polityczna produktywność.

Die Geschichte von Sozialpädagogik und Sozialarbeit ${ }^{2}$, nunmehr seit etwa 35 Jahren ,Soziale Arbeit' genannt, in Deutschland ist in die Gesellschaftsgeschichte der deutschen Länder seit den Zeiten des ,Alten Reiches' (Heiliges Römisches Reich Deutscher Nation) bis zur Bundesrepublik Deutschland einzubetten ${ }^{3}$. Dabei gilt insbesondere für die Soziale Arbeit, was Hans-Ulrich Wehler als entscheidendes Prinzip einer gesellschaftsgeschichtlichen Vorgehensweise herausstellt:

Die Entwicklung auch der deutschen Gesellschaft in den vergangenen beiden Jahrhunderten ist durch die Dauerhaftigkeit struktureller sozialer Ungleichheit grundlegend bestimmt. Sie ist vielleicht die Hauptfrage jeder historischen Sozialforschung, jeder Stratifikationsanalyse, jeder Sozialgeschichte - nicht zuletzt auch deshalb, weil damit die Lebenschancen der vielen Individuen notwendig in den Blick kommen (Wehler 1987: 16) ${ }^{4}$.

\footnotetext{
2 Die deutsche Besonderheit besteht darin, dass im Kontext wohlfahrtstaatlicher Regulierungen und Leistungen seit langem - mehrheitlich und nur noch selten umkämpft - nicht mehr zwischen Sozialpädagogik und Sozialarbeit getrennt wird. Dies erfordert nichts desto trotz von Zeit zu Zeit Reflexionen über beides, so dass in diesem Text an einigen Stellen nicht von Sozialer Arbeit, sondern von Sozialpädagogik bzw. Sozialarbeit gehandelt und gesprochen wird. Zum Stand der Sozialpädagogik schreibt M. Winkler im ersten Absatz seiner großformatigen Studie „Eine Theorie der Sozialpädagogik“: „Die Theorie der Sozialpädagogik ist ein schwieriges Geschäft, das sich nicht von selbst versteht. Ihr Anliegen kann kaum auf unbedingte Zustimmung hoffen, noch findet es Voraussetzungen, die als hinreichend gesichert gelten. Damit ist zwar kein neuer Sachverhalt genannt, denn der Streit um die Sozialpädagogik und ihr angemessenes begriffliches Verständnis ist nahezu so alt wie der Terminus selbst und die Beschäftigung mit dem Thema. Aber in der eigentümlichen Gleichzeitigkeit von Tendenzen der Modernisierung und Demodernisierung, welche wenigstens die fortgeschrittenen Staaten und Gesellschaften gegenwärtig auszeichnen, hat das Nachdenken über Sozialpädagogik an Brisanz gewonnen. Mehr noch als das: Sozialpädagogik ist ein prekäres Thema geworden“ (Winkler 1988: 11).

${ }^{3}$ Dies ist der Gegenstand der 5 Bände „Deutsche Gesellschaftsgeschichte“ von Wehler. Wir sehen hier ab von den Besonderheiten der Entwicklung in der DDR, die eine eigene Studie benötigte.

${ }^{4}$ Diese These von der Kontinuität sozialer Ungleichheit wird auch von der neuesten Sozialstrukturanalyse Deutschlands bestätigt. Diese grundlegende Analyse klassenbasierter sozialer Milieus zeigt ,Oben Mitte - Unten' in ihrer gegenwärtigen Differenziertheit und Verteilung auf verschiedene Klassenfraktionen mit Konsequenzen für Gesellschaftsbild, politisches Bewusstsein und Selbstverständnis (Vester et al. 2001).
} 
sozialen Ungleichheit komme in jeder Gesellschaft eine hervorragende Bedeutung zu, so dass es berechtigt erscheine, dieses System als eine der Zentralachsen von Gesellschaftsanalyse zu behandeln.

Man muß sich jedoch klarmachen oder dessen bewußt bleiben, daß soziale Ungleichheit (...) strenggenommen ein Ergebnis des Zusammenwirkens von ungleicher Macht- und Herrschaftsverteilung, ökonomischer Lage und kulturellen Entwürfen der Weltdeutung darstellt. Deshalb bleibt sie ein Resultat der Überschneidung der drei systematisch vorgeordneten Dimensionen (Wirtschaft, Herrschaft und Kultur, die Autoren) (Wehler 1987: 11).

Eingebunden in die Analyse sozialer Ungleichheit ist zugleich die Frage nach Prozessen der Klassenbildung, die es jeweils historisch-systematisch zu entziffern gilt (s. Kocka 1990a, b). Dies hat es Lenhardt und Offe (1977) vor langer Zeit erlaubt - bezogen auf den Bereich der Sozialpolitik - ihre „Proletarisierungsthese“ vorzutragen, die „die staatliche Bearbeitung des Problems der dauerhaften Transformation von Nicht-Lohnarbeitern in Lohnarbeiter" (ibidem: 101) zum Gegenstand hat und darin unmittelbar relevant für Soziale Arbeit ist.

In der rekonstruktiven Betrachtung zeigt sich, dass die Entwicklung der Disziplin und Profession Sozialer Arbeit in deutschen Landen wie im europäischen Raum insgesamt mit der Geschichte von Armenfürsorge und deren Institutionalisierung beginnt. In den spätmittelalterlichen Städten (in Deutschland zu Beginn des 16. Jahrhunderts) bildeten sich zwar schon zwei elementare Institutionen heraus, das Hospital und die Bettel-, Armen- und Almosenordnungen, die als Strategien zur Bearbeitung sozialer Notlagen entwickelt und eingesetzt wurden. Bis weit in das 16. Jahrhundert hinein folgte jedoch in vielen ländlichen Gebieten die Mittelvergabe an Arme weder einer gesellschaftlichen Logik, noch unterlag sie dem Primat der Rationalität: Dieses Geben war an der Norm religiöser Mildtätigkeit/Nächstenliebe orientiert. Die wesentliche Begründung lag allerdings darin, dass sich reiche Spender durch mildtätige Gaben an die Armen ihr „Seelenheil“ zu erkaufen hofften (vgl. Sachße, Tennstedt 1980: 23, 29f.; Mollat 1987). Die Etablierung und Aufrechterhaltung der städtischen Institutionen, die ein mehr oder weniger konsistentes Armenrecht - gestützt auf Kommunalisierung, Bürokratisierung und Pädagogisierung - implementierten, kam erst durch herrschaftliche Interessen und Interventionen im Rahmen der Etablierung und Durchsetzung des Kapitalismus zustande (Sachße, Tennstedt 1980: 34). Das Voranschreiten gesellschaftlicher Arbeitsteilung in handwerklichen Betrieben, die Ausweitung des Handels und die zunehmende Warenzirkulation unterschiedlicher Erzeugnisse begünstigte vielgestaltige dörfliche und städtische Lebensformen, die auf eine Verwertung der Ware Arbeitskraft angewiesen waren (vgl. Marx, o. J.: 375-413). 
Herrschaftsträger kontrollierten die wirtschaftlichen Abläufe, entzogen sich aber gleichzeitig der gesellschaftlichen Kontrolle zur Regulierung der von ihnen verursachten ökonomischen und sozialen Notlagen. Die rechtlichen Verfahren und die Handlungskonzepte der öffentlichen Einrichtungen gingen nicht immer konform mit den gesellschaftlichen und kulturellen Wahrnehmungs- und Deutungsmustern von Armut, d.h. die „moralische Ökonomie“ der Mehrheit wirkte zu Beginn der Änderungen des Bettel- und Almosenrechts auf Seiten der nunmehr zu Disziplinierenden oder Verfolgten (vgl. Sachße, Tennstedt 1980: 38). Wichtigstes Element der herrschaftlichen Inklusions- bzw. Exklusionssemantiken war die Einführung bürokratischer Institutionen, die objektivierte, d.h. mit öffentlicher Autorität versehene, Klassifizierungen etwa als Bettelvögte durchsetzten und diese auf Gruppen bzw. Individuen übertrugen; entscheidendes Kriterium wurde - für die bürgerlich-kapitalistische Gesellschaft durchgehend bis heute - die Bereitschaft, Arbeitswilligkeit zu demonstrieren. Die Setzung von autoritativen Grenzen zwischen den Gruppen, in Form von symbolischen Ordnungen und kollektiven Repräsentationen, wiesen den Armen einen legitimen oder illegitimen Platz in der sozialen Lebenswelt zu. Das Hinwirken auf Veränderungen in den weltanschaulichreligiösen Deutungsmustern und die bewusste Ablehnung bzw. der Entzug aus dem kontrollierenden und gestaltenden Zugriff der Herrschenden waren die einzigen Mittel der Gruppen von Armen, den mit der sozialen Fürsorge einhergehenden repressiven Maßnahmen zu entgehen (vgl. Raphael 2004: 15f.; Geremek 1988: 300).

Mit dem Übergang vom Feudalismus zum Kapitalismus etabliert sich dementsprechend ein neuer Modus von Vergesellschaftung, mit dem - formal - die für vorkapitalistische Gesellschaftsformationen bestimmende persönliche Abhängigkeit durch ein System persönlicher Unabhängigkeit, wie Marx es nennt, gegründet auf sachlicher Abhängigkeit beginnt (Marx o. J.: 75). Dies bedeutet die Existenz als Arbeitskraftbesitzer, dem die Gestalt „Kapitalist“ gegenübertritt. Daraus erwachsen dann historisch konkret immer wieder allgemeine wie besondere Widersprüche zwischen Individuum und Gesellschaft, die zur ,Einrichtung' von Sozialpädagogik führt ${ }^{5}$. „Kurz: Sozialpädagogik hat die Aufgabe, die Risiken zu bearbeiten, welche in kapitalistischen Marktwirtschaften entstehen“ (Winkler 1995: 173; vgl. Sünker 1995a).

Soziale Arbeit ist mithin im Widerstreit von Gesellschaft und Individuum zu lokalisieren, fungiert über weite Strecken in den historischen Prozessen bis heute als Instanz, gesellschaftliche Normalität durchzusetzen, sie wird daher gesellschaftspolitisch mehrheitlich instrumentalisiert - und hat sich bis heute als funktional für die Reproduktion der Gesellschaft, wie sie ist, erwiesen. Denn Abweichung wird im Rahmen einer Pädagogisierung des Politischen und Gesellschaftlichen als individuelles Problem definiert (Sünker 1995b).

\footnotetext{
${ }^{5}$ So klassisch 1959 in der Dissertation von K. Mollenhauer, der „Ursprungsstudie“.
} 
Historisch betrachtet lassen sich dementsprechend Keime der Zugriffsbewegungen auf Individualität, die für die Gegenwart häufig aus einer FoucaultPerspektive als neu konstatiert werden, bereits in der frühbürgerlichen Gesellschaft auffinden. Dass es sich hier um ein systemisch induziertes Problem handelt, wird bereits anhand der Darstellung der Widersprüche der bürgerlichen Gesellschaft in der Hegelschen "Philosophie des Rechts“ deutlich. Hier wird die Frage nach dem Vergesellschaftungsmuster in den Folgen für die Existenz und Verfassung der Mitglieder der bürgerlichen Gesellschaft thematisch. Es geht um Form und Gehalt der Beziehung der Mitglieder der bürgerlich kapitalistischen Gesellschaftsformation aufeinander, was einen spezifischen Ausdruck in deren Selbstständigkeit als Einzelne findet. Der darin zugleich seinen Ausdruck findende äußerliche Zusammenhang der selbstständigen Einzelnen miteinander (vgl. Hegel 1955: §§ 182 und 183) führt wegen dieser Voraussetzungen zu strukturellen wie handlungspraktischen Widersprüchen: „Die bürgerliche Gesellschaft bietet in diesen Gegensätzen und ihrer Verwickelung das Schauspiel ebenso der Ausschweifung, des Elends und des beiden gemeinschaftlichen physischen und sittlichen Verderbens dar" (Hegel 1955: 185).

Eingebunden in diese Darstellung der Realität der bürgerlichen Gesellschaft, zu der Hegel die Lektüre der schottischen Moralphilosophen geführt hatte, ist der Aufweis einer gesellschaftlich notwendigen, weil ihrem Charakter entsprechenden Entwicklung: Diese beinhaltet eine „unendliche Vermehrung der Abhängigkeit und Not“ (ibidem: § 195), das „Herabsinken einer großen Masse unter das Maß einer gewissen Subsistenzweise“ - was Armut bzw. Pauperisierung als Massenerscheinung zur Folge hat -, und „zugleich die größere Leichtigkeit, unverhältnismäßige Reichtümer in wenige Hände zu konzentrieren, mit sich führt“ ( Hegel 1955: § 244).

Zugleich weist Hegel darauf hin, dass Armut gegen das Gefühl der Ehre, sich ohne Hilfe anderer reproduzieren zu können, verstößt. Damit ist die regulative Idee der bürgerlich kapitalistischen Gesellschaftsformationen - auf die hin Soziale Arbeit orientieren soll - benannt.

Mollenhauer hat in seiner Studie über den Ursprung der Sozialpädagogik in Deutschland darauf aufmerksam gemacht, dass Sozialpädagogik in ihren Anfängen die eigene Entstehung und Perspektive dadurch bestimmt, dass sie die Situation der bürgerlichen Gesellschaft durch „Kulturverfall“ gekennzeichnet sieht. Daraus folgt die berufliche Aufgabe „einer umfassenden sozialen Regeneration“, die „das Volksganze zum Gegenstand der Erziehung“ macht (Mollenhauer 1959: 121-122).

Sozialpädagogik verfehlte also von Anbeginn an ihren Gegenstand, indem sie sich auf vermeintliche, vormoderne Vergemeinschaftungsformen und die „Erneuerung des Volkslebens“ (Mollenhauer 1959: 122) bezieht, während - wie die Hegelsche Analyse zeigt - die bürgerliche Gesellschaft als Gesellschaft stetig ihre eigenen Widersprüche und Spannungsverhältnisse produziert. Aus diesem Fehler erwächst der individualisierende Zugriff sozialpädagogischen Handelns, die Normalisierungsarbeit, auf Einzelne, Gruppen, Klassen - sei es legitimiert durch Philanthropie, ,moral panic' oder die Rede von den gefährlichen Klassen. 


\section{Armenpflege und Kapitalismus}

Die Armenpolitik im 19. Jahrhunderts ${ }^{6}$ war durchzogen von Kontrolle und Disziplinierung der Armen und der Vergabe von Leistungen, die an individuelle Bedürftigkeitsprüfungen und Interventionsstrategien gekoppelt waren und sowohl über innere als auch äußere Zwänge organisierter Normalitätskontrolle gesteuert wurden - dies nicht nur marginal mit Hilfe von Arbeits - und Zuchthäusern. Es gab kommunale und länderspezifische Regularien, die in ihrer jeweiligen Ausformung sehr unterschiedlich sein konnten. Allgemeingültige Richtlinien, Vorschriften oder Gesetze wurden jedoch nicht erlassen. In der Regel waren kirchlich-karitative und private Einrichtungen für armenpflegerische und fürsorgerische Leistungen zuständig. Politisch ging es vorrangig nur um Schutz und die Sicherheit der herrschenden Klasse vor der Masse der Verarmten, das Wohlergehen der Arbeiter spielte eine untergeordnete Rolle. Die Entwicklung von Sozialreformen, die konkrete sozialpolitische Forderungen entwickelten und politisch durchsetzen konnten, führte zur Etablierung neuer Gesetzgebungen ab 1842, die im gesamten Deutschen Reich umgesetzt wurden (vgl. Salomon [1921] 2004: 106ff).

Nimmt man derartige Vorstellungen als wünschenswerten Maßstab, war die Entwicklung der i.d.R. konfessionell geprägten karitativen Einrichtungen der Kinder-, Jugend-, Familien- und Krankenfürsorge und ebenso die halböffentlichen kommunalen Initiativen des Elberfelder und später des Straßburger Modells, die mit den Hauptaufgaben der Armenfürsorge betraut waren, ein notwendiger weiterer Schritt. Diese Einrichtungen blieben den traditionell christlich paternalistischen Leitbildern verhaftet, d. h. sie verbanden ein Verständnis von Nächstenliebe mit der Vorstellung einer „gerechten“ väterlichen Gewalt, einer Erziehungsvollmacht zu jenem sozialen Verhalten, das innerhalb der nicht-armen, bürgerlich geprägten Normalgesellschaft als erstrebenswert und erfolgreich galt. Unter dem Einfluss malthusianischer Ideen wurde die Armenpflege auf das notwendigste beschränkt, jede erzieherische Intervention wurde beiseitegelassen und selbst Kinder nur nach den Prinzipien der Armenpflege unterstützt (Bühler-Niederberger, Sünker 2014). Das war ein Rückfall hinter die Errungenschaften der Hamburgerischen Armenanstalt des 18. Jahrhunderts (vgl. Salomon [1921] 2004: 113). Die anfänglich sehr bescheidenen Schutzregelungen im Bereich der Regulierungen von Arbeitszeiten, Schutzmaßnahmen bei gesundheitsschädigenden Arbeiten und Unfällen führten gleichzeitig - vor allem in großen Städten - zur Neuregelung der Armenpflege in

\footnotetext{
${ }^{6}$ Steinmetz (1993) hält für den gesellschaftsgeschichtlichen Analyserahmen fest: „The pre-1914 German welfare state therefore allows one to study the simultaneous existence of at least four distinct types of social policy: poor relief, 'Bismarckian' social insurance, proto-corporatist policies, and modern social work. Although these do not exhaust the full universe of forms of social policy, they represent four distinct and extremely influential strategies for regulating 'the social'. More recent social programs have often operated within the traditions, the specific categories, technologies, and goals, established by these early forms"(ibidem: 5).
} 
kommunalen Armenbezirken, wie bspw. im Elberfelder System, mit ehrenamtlichen männlichen Pflegern und Vorstehern (vgl. Sachße, Tennstedt 1986: 31; Salomon ([1901] 1997): 90f.).

Dabei wurden mehrere Ziele miteinander verknüpft. Vorrangig ging es um die „Zivilisierung“ der Klasse der Unterschicht im allgemeinen, darüber hinaus um die Regulierung des beunruhigenden und gesellschaftsgefährdenden Potentials der unterprivilegierten Massen und um die Reform unzureichender privater Wohltätigkeit durch den Einsatz von effektiveren Staatshilfen - dies zum Zwecke von Sozialkontrolle durch Sozialdisziplinierung sowie der Produktion von kampf- und arbeitsfähigen Menschen. Getragen wurden diese Veränderungsprozesse von Vorstellungen der generellen Ausdehnung pädagogischer Grund- und Geisteshaltungen als auch dem Glauben an die modernen Humanwissenschaften, die Theorien zur Abschaffung von Krankheit, Elend und Kriminalität entwickelten. Durch die Einführung des preußischen Zwangserziehungsgesetzes von 1878, in dem für Kinder unter 12 Jahren Erziehungsmaßnahmen festgeschrieben wurden, die anstelle von Strafen zur Anwendung kamen, wurden diese Vorstellungen gesetzlich gerahmt. Damit konnte an der Nahtstelle zwischen Vormundschafts- und Strafrecht eine besondere Sphäre der Zwangserziehung eingeführt werden. Der zweite Aspekt betraf das staatliche Einwirken auf die Freizeitgestaltung der Jugendlichen, welches u. a. mit der Entwicklung öffentlicher Angebote zur Freizeitgestaltung der Jugendlichen eingeleitet wurde. Diese Erziehungshilfen wurden von vaterländischen und paramilitärischen Vorstellungen stark beeinflusst und grenzten sich klar von autonomen Gruppen ab, wie beispielsweise der Wandervogelbewegung oder der sozialistischen Arbeiterjugend (Peukert 1989: 311).

Erst der zunehmende Personalbedarf dieser neuen Systeme wirkte sich begünstigend auf die weiblichen Bestrebungen aus, als Armenpflegerinnen zu arbeiten. Die männlichen Armenpfleger organisierten einen massiven Widerstand, da sie die Arbeit von Frauen - im Gegensatz zur interessierten Fachöffentlichkeit als nicht notwendig und als unprofessionell aburteilten. Ab dem Jahr 1881 begannen einzelne Stadtverwaltungen - wie bspw. Kassel damit - Frauen als gleichberechtigte Armenpflegerinnen einzusetzen; es folgten Berlin, Frankfurt, Düsseldorf, Wiesbaden, Magdeburg. Im Jahr 1907 waren in Berlin 4.000 männliche Pfleger und 40 Pflegerinnen beschäftigt (vgl. ausführlich Salomon [1901] 1997: 93; Sachße, Tennstedt 1995: 214f.).

Zeitgeschichtlich verknüpft ist die Entstehung von Sozialer Arbeit als Profession mit der Geschichte des Vereins der „Mädchen- und Frauengruppen für soziale Hilfsarbeit" (Salomon 1913). Dieser Verein ist aufs engste mit der beruflichen Karriere und der Einflussnahme von Alice Salomon auf die Herausbildung der Sozialen Arbeit als Profession verbunden. Sie war dort und in der „Auskunftsstelle der Deutschen Gesellschaft für ethische Kultur", der späteren Centrale für private Fürsorge, über mehr als ein Jahrzehnt intensiv in der ehrenamtlichen Fürsorgearbeit tätig. 
Die anfängliche Ausrichtung der Mädchen- und Frauengruppen für soziale Hilfsarbeit - getragen durch die sozialen Vorstellungen breiter philanthropischer Kreise - auf die Gewinnung und Schulung von Kräften für die ehrenamtliche Soziale Arbeit veränderte sich im Kontext der Bildungsreformen schnell zu einer Vorstellung von Sozialer Arbeit als professionellem Frauenberuf (vgl. Salomon ([1918] 2000): 495). Die ersten sozialwissenschaftlich orientierten Lehrgänge weiteten sich ab 1899 zu Jahreskursen und Ferienkursen für Berufsarbeit in der Wohlfahrtspflege sowie zu Vortragsreisen aus und führten ab 1905 zur Gründung von Frauenschulen. Die maßgeblichen Impulse für diese Gründungswelle in der Zeit vom 1909-1914 kamen von Vereinen in Form der Mädchen- und Frauengruppen für soziale Hilfsarbeit, aber auch Bildungsvereinen, Stiftungen und Kuratorien, da Wohlfahrtsarbeit am Anfang des 20. Jahrhunderts noch in großem Umfang freie Vereinsarbeit war. Diese neu gegründeten Schulen wurden größtenteils von konfessionellen Trägern finanziert, wie bspw. die Frauenschule der Inneren Mission in Berlin (1909), die soziale und karitative Frauenschule in München (1909) oder das Evangelisch-Soziale Frauenseminar in Elberfeld (1910). Die Gründung der nichtkonfessionell ausgerichteten Sozialen Frauenschule 1908 in Berlin durch Alice Salomon wurde ermöglicht aufgrund ihrer Einbindung in das nationale und internationale Netzwerk der bürgerlichen Frauenbewegung, welches von ihr mit gestaltet wurde. Das Zentrum bildeten die Mädchen- und Frauengruppen für soziale Hilfsarbeit, deren Vorsitzende sie nach dem Tod von Jeanette Schwerin wurde. Dieses Netzwerk verband WissenschaftlerInnen mit PraktikerInnen städtischer wie privater Wohlfahrtseinrichtungen und PolitikerInnen und war international ausgerichtet. Durch die professionelle Soziale Arbeit sollte eine Einbindung der bürgerlichen Mädchen und Frauen in die soziale Hilfsarbeit stattfinden, um sie qualifiziert auf die verantwortungsvolle Tätigkeit in der Sozialen Arbeit vorzubereiten und gesellschaftliche Reformen mitzugestalten. Es entwickelte sich Soziale Arbeit als Disziplin, als Ausbildungs- und Studiengang, welche zur Gründung einer Vielzahl von Schulen, Akademien und schließlich Hochschulen führte sowie zu nationalen und internationalen Vereinigungen, wie der Konferenz Sozialer Frauenschulen Deutschlands und des Internationalen Komitees Sozialer Schulen. Die Schule selbst war Ausgangspunkt konzeptioneller Initiativen; hier konnten die SchülerInnen Klassen- und Standesgrenzen überschreiten, indem sie diskursive Auseinandersetzungen um gesellschaftliche Normen und Werte führten. Ziel war es, ein neues Bildungsbewusstsein zu entwickeln.

Begleitet wurden diese Veränderungen durch eine Vielzahl von neuen gesetzlichen Regelungen, wie bspw. 1923 dem Reichsjugendwohlfahrtsgesetz (RJWG). Zwar stellt das RJWG leitmotivisch das Recht des Kindes auf Erziehung in den gesetzlichen Fokus, in seiner Ausrichtung ging es jedoch nicht um die Entfaltung der Einzelpersönlichkeit als Individualrecht, sondern um das Recht des Staates als Kontrollinstanz in das Leben der Kinder und Jugendlichen einzugreifen - dies durch 
Korrekturen der Erziehungsprozesse. Damit wurde ein sozialpädagogisches Feld konstituiert, das bis heute die charakteristische Vernetzung von sozialer Hilfe und Kontrolle aufweist und neben fördernden eben auch restriktiv korrigierende Maßnahmen beinhaltet, die sich an diffusen gesellschaftlichen Normalitätsidealen orientierten und begleitet wurden von Debatten über die „Grenzen der Erziehbarkeit" (vgl. Peukert 1986: 321 f.) ${ }^{7}$. Die ältere Rettungsarbeit ${ }^{8}$ - hier insbesondere im Kontext der Erziehung von Kindern und Jugendlichen - und die fürsorgerische Wohltätigkeit wurde mit pädagogischen Staatsinterventionen in private Erziehungsprozesse gekoppelt und durch die Schaffung neuer behördlicher Einrichtungen - den Jugendämtern - institutionalisiert und professionalisiert (vgl. Peukert 1989: 313 f. $)^{9}$.

In seiner grundlegenden Studie „Grenzen der Sozialdisziplinierung. Aufstieg und Krise der deutschen Jugendfürsorge 1878-1932“ hat Peukert (1986) diesen Prozess historisch präzise rekonstruiert. Er analysiert die Geschichte der Institutionalisierung eines pädagogischen Handlungsfeldes im Kontext der Durchsetzung der bürgerlich-kapitalistischen Gesellschaft in Deutschland. Er unternimmt es, mithilfe eines sozialkulturellen Ansatzes das Beispiel "Jugendfürsorge“ für die Analyse gesellschaftlicher und pädagogischer Entwicklung fruchtbar zu machen, indem er drei historisch-systematische Problemstellungen und deren Vermittlung auseinander legt: a) Geschichte der Jugend; b) Geschichte der Sozialpädagogik; c) Bedingungen, Möglichkeiten und Grenzen pädagogischen Fortschritts. Zu diesem Zweck rekonstruiert er Voraussetzungen und Konsequenzen gesellschaftlicher Prozesse wie pädagogischer Diskussionen und Konzeptentwicklungen.

Neben der spannend zu lesenden Ausbreitung des historischen Materials für die Zeit zwischen 1878 und 1932 finden sich in dieser Studie wesentliche Beiträge zur Theoriedebatte: die Pädagogisierung sozialer Probleme mit dem Anschein

\footnotetext{
7 Dass das „Bewahrungsgesetz“ letztlich nicht verabschiedet wurde, obwohl die Sozialpädagogen es propagierten und es den nationalsozialistischen Vorstellungen entsprach, folgt für Peukert aus einem ,Kompetenzgerangel‘ zwischen Fürsorge, Justiz und Polizei (Peukert 1989: 331).

8 Der Hamburger Theologe Johann Hinrich Wichern gilt als Mitbegründer des Rauhen Hauses bei Hamburg, des Berliner Johannesstifts und der Inneren Mission. Durch Wichern wurde die Jugendwohlfahrtsarbeit im Kontext eines zutiefst christlichen Verständnisses von Fürsorge in Rettungsanstalten institutionalisiert; durch äußere Missionstätigkeiten sollten die sozialen und sittlichen Verhältnisse in der Gesellschaft stabilisiert werden (vgl. Richter 2004).

${ }^{9}$ Damit verbindet sich - nicht nur - für Peukert „die Pädagogisierung des Wohlfahrtswesens“: „Dem Anspruch nach bezweckte alle neuzeitliche Wohlfahrtstätigkeit neben der Linderung aktueller Notlagen immer die Erziehung der Betroffenen zur Selbst-Subsistenz, zum Leben in ehrbarer Armut, allemal besonders zu pflichtbewusster Arbeitsleistung. Negativ diente dazu die restriktive Festsetzung von Art und Ausmaß der Hilfe auf ein Minimum, das sich unterhalb dessen bewegen sollte, was mit der schlechtestentlohnten ,freien' Arbeit zu erzielen war. Positiv sollten neben dieser Nötigung zur Lohnarbeit aber doch das Angebot an Arbeitsmöglichkeiten und die erzieherische Gewöhnung an disziplinierte Leistung stehen“ (Peukert 1989: 320). Diesem Leitmotiv entspricht auch die Einschätzung von Steinmetz, der unter der Überschrift „Scientific Social Work“ für die Entwicklung nach 1890 festhält: „Pedagogic methods therefore became central“" (Steinmetz 1993: 199).
} 
sozialer Hilfe bedeutet in der Realität des hier untersuchten Feldes sowohl eine repressive soziale Normierung als auch die Politik der Ausgrenzung von Randschichten. Sozialpädagogik ist daher mit Problemen befasst, die außerhalb ihrer Reichweite permanent neu entstehen. Entscheidend aber ist die These von Peukert, dass der Vision einer lückenlosen Sozialkontrolle die Realität des kapitalistischen Reproduktionsprozesses gegenübersteht, innerhalb dessen immer wieder neue Kontrolllücken - aus strukturellen Gründen - entstehen ${ }^{10}$.

\section{Weimarer Republik und Nationalsozialismus}

Die Einleitung von Reformbestrebungen wurde überlagert von der allgemeinen Krise der Sozialpädagogik, die durch die Krise in der Endphase des Weimarer Sozialstaates induziert war (1929-1932) und sich in der mangelnden Finanzierbarkeit der öffentlichen Hilfen und in den Skandalen der geschlossenen Fürsorge als auch in Problemen in der offenen Jugendarbeit offenbarte. Vermehrt kam es zu der - in hegemonialen Auseinandersetzungen formulierten - Feststellung, dass sich Teile der Unterschicht (sjugend) nicht disziplinieren ließen. Die Folgen waren einerseits eine selbstkritische Revision der traditionellen Anstaltspädagogik als auch der liberalen Methodik in den praktischen Handlungskonzepten der Sozialen Arbeit, aber andererseits auch disziplinäre und professionelle Debatten in der Fürsorgeerziehung um die „Grenzen der Erziehbarkeit“, die in Nazi Deutschland in der Einrichtung von Jugendkonzentrationslagern endeten (Peukert 1989: 323, 331). Neben der Selbstkritik in der Sozialpädagogik entstanden vermehrt Forderungen nach der Ausgrenzung von „Unerziehbaren“, die durch rassebiologische Ideologien gestützt wurden. Es wurde ein Auslesediskurs geführt, der sich 1932 in der RJWG-Novellierung niederschlug. Diese erste Einführung der Unterscheidung in „Erziehungswürdige“ und „Unverbesserliche“ führte dann im Nationalsozialismus rassebiologisch überhöht zu der Auslese- und Ausmerze-Dichotomie und wurde mit Bedenkenlosigkeit praktiziert (vgl. Peukert 1989: 332f.). Im Nationalsozialismus - der deutschen Form des Faschismus - wurden die Institutionen der Sozialen Arbeit - im Kontext der Entwicklung des Wohlfahrtsstaates zu einem Erziehungsstaat - zu Regulationsinstanzen besonderer Art (vgl. Schnurr 1988)11. Erziehung als wesentlicher Bestandteil der Sozialen Arbeit richtete sich mehr und mehr an der Ideologie der Volksgemeinschaft aus und wurde von einem System sozialer Kontrolle eingefasst.

\footnotetext{
10 Siehe zur Analyse dieses Feldes auch die großformatige Studie von Steinacker (2007) „Der Staat als Erzieher".

11 Schnurrs (1997) historische Analyse der sozialpädagogischen Bewegungen im Nationalsozialismus zeigt divergierende Handlungskonzepte der Sozialarbeiter in dieser Zeit auf, die von Teilhabe bis hin zu Fehlinterpretationen, passiver als auch aktiver Integration in das System der Volkswohlfahrt gehen.
} 
Die Ideologie der Volksgemeinschaft (Sünker 2006a) war fundierend für die politische Kultur und die Praxis des Nationalsozialismus in allen gesellschaftlichen Bereichen und steuerte über den Begriff der „Gemeinschaftsfähigkeit“ die Prozesse von Integration und Ausgrenzung in sozialarbeiterischen Kontexten. Erbbiologisch und rassehygienisch konkretisierten sich diese Prozesse als Kontrolle, Verfügbarmachung, Ausmerze, Verfolgung und Mord. Die Ideologie der Volksgemeinschaft bot Orientierung für das individuelle Bewusstsein als auch die sozialen Beziehungen, die sich in den Institutionen der sozialen Arbeit zu der nationalsozialistischen „Volkswohlfahrt“ entwickelten und dazu führten, dass das sozialarbeiterische Alltagshandeln durch eugenische und rassistische Vorstellungen bestimmt wurde. Die Grundlage hierfür bildeten eine Zerstörung wohlfahrtsstaatlicher und hilfepolitischer Diskurse, mit der der Nutzen des/der Einzelnen für die Volksgemeinschaft, d.h. Willfährigkeit und Einsatzwille, zum Maßstab für den Zugang zu oder den Ausschluss von bestimmten sozialen Unterstützungen wurde ${ }^{12}$.

In der Mischung von „Fürsorge und Unterdrückung“, einem „fürsorglichen Gehabe“ des Nationalsozialismus gehen Integration und Ausgrenzung, Strategien zur „Bändigung der deutschen Arbeiterklasse“ zusammen, um die Volksgemeinschaft als totale „Leistungsgemeinschaft“, so die Analyse Masons, durchzusetzen - KostenNutzen-Rechnungen kennzeichnen Ideologie und Praxis. Soziale Arbeit - mit ihren vielfältigen Aktionen und Institutionen - als Instrument der Herrschaftssicherung des Nationalsozialismus lebte von der Suggestion alltäglicher Normalität gegen die Realität des alltäglichen Terrorismus, war ausgerichtet auf die ,Gesunderhaltung des deutschen Volkes zur Entwicklung militärischer Stärke, um im sozialdarwinistischen Kampf zu siegen (Sünker 1991).

\section{Post-Faschismus: Theorieentwicklungen und Professionalisierungsprozesse}

Vor dem Hintergrund der deutschen Spaltung, Kontinuitäten und Brüchen mit dem Nationalsozialismus beginnt in der Bundesrepublik Deutschland der Versuch, im Kontext der Rekonstruktion des Kapitalismus, einen Sozialstaat aufzubauen. Dies geschieht selbstverständlich zum einen, um die Legitimität der bürgerlichkapitalistischen Gesellschaftsformation herauszustellen und zum anderen Massenloyalität zu sichern.

Dementsprechend geht es von Anfang an immer um die Frage, was Sozialstaatlichkeit tatsächlich bedeutet. Die Alternativen hat Hartwig (1977: 12; vgl. 54, 283, 314) systematisch vorgestellt:

\footnotetext{
12 Wichtig ist darüberhinaus, dass die Vertreter geisteswissenschaftlicher Pädagogik und Sozialpädagogik - wie vor allem H. Nohl - sich in der Zeit des Nationalsozialismus nicht als widerständig, sondern als systemkonform erwiesen; s. dazu Ortmeyer 2009).
} 
Modellartig stilisiert liegt die grundlegende Alternative a) in einer Verwirklichung von Sozialstaatlichkeit auf der Basis der gegebenen Besitz- und Statusverhältnisse mit sozialpolitischen Korrekturen und Ausgleichsinterventionen für alle Teile der Gesellschaft, b) in der Veränderung der überkommenen Besitz- und Statusverhältnisse mit den Mitteln des demokratisch strukturierten Staates, um den in der Verfassung verankerten Rechten, vor allem denen der Freiheit und Gleichheit zu gesellschaftlich realer Geltung zu verhelfen. Hier liegt der Schlüssel für die Deutung des Sozialstaatsgrundsatzes der Verfassung (ibidem: 12).

Die Frage der Demokratisierung ist - wie nicht anders zu erwarten - im Kontext von 1968 als der Signatur einer Epoche auch für die Soziale Arbeit bedeutsam geworden: Ähnlich wie in Großbritannien und den USA hat sich auch in der BRD eine ,Politisierung' Sozialer Arbeit, d.h. die Erkenntnis ihrer Instrumentalisierung für die Zwecke der Herrschenden verbreitet und zur Frage nach gesellschaftlichen Alternativen wie Folgen für die Praxis ergeben (vgl. Sünker 2003; Steinacker, Sünker 2010).

Mit diesen Entwicklungen ist zugleich der entscheidende Bezugspunkt für Selbstverständnis wie Perspektiven der Grundlegung von Sozialer Arbeit für die Gegenwart benannt. Zudem beinhaltet dieser Bezug die Möglichkeit, die gesellschaftliche wie wissenschaftliche Verortung von Sozialpädagogik wie Sozialarbeit in der BRD nachvollziehen zu können: Während die Sozialpädagogik in der Kontinuität ihrer universitären Präsenz seit den Weimarer Zeiten an ausgewählten bundesdeutschen Universitäten beheimatet blieb, musste sich die Sozialarbeit erst einmal „hocharbeiten“ - vom Fachschulniveau über die Höhere Fachschule für Sozialarbeit gelang ihr - zeitgleich mit einem massenhaften Ausbau und einem Professionalisierungsprozess - der Aufstieg auf das Fachhochschulniveau. Universitär bedeutsam wurde die Sozialarbeit gleichwohl nur im Zusammenspiel mit der Sozialpädagogik eben im Kontext der Schrägstrich-Konzeption oder unter dem Namen „Soziale Arbeit"13.

Die Arbeit an der Theoriebildung soll hier mit Bezug auf einige ausgewählte Beispiele skizziert werden ${ }^{14}$. Klaus Mollenhauers (1959) Arbeiten bestimmen über weite Strecken die Entwicklung und Institutionalisierung der Sozialen Arbeit in der BRD - zum einen mit seiner Dissertation Ursprünge der Sozialpädagogik in der industriellen Gesellschaft (1959), zum anderen durch seinen Übergang von der

\footnotetext{
${ }^{13}$ Es ist hier nicht der Platz, um die vielfältigen Beiträge zur Professionalisierungsdebatte zu rekonstruieren, vgl. exemplarisch für den Anfang im Rahmen einer sozialwissenschaftlich Wende Otto (1973). Der Prozess von Disziplin-wie Professionsbildung wird zudem wesentlich erkennbar in voluminösen Produkten wie dem - mehrfach neu aufgelegten - Handbuch Soziale Arbeit (Otto, Thiersch 2014) oder dem „Grundriss Soziale Arbeit“ (Thole 2005).

14 Dabei ist entscheidend, dass für die Zeit der Theoriefundierung drei „Gründungsväter" (Mollenhauer, Thiersch, Otto) mit ihren jeweils sehr unterschiedlich akzentuierten Arbeiten und ihren Schulen wesentlich waren; vgl. Füssenhäuser (2005).
} 
geisteswissenschaftlichen Pädagogik zu einer mehr sozialwissenschaftlichen Orientierung. Er entwickelt schon früh aus einer hermeneutisch-praktischen Tradition der Erziehungswissenschaft heraus ein Theorie-Praxis-Verständnis, das die Trennung von Praxis- und Wissenschaftswissen einfordert. Seine Arbeiten zur Allgemeinen Pädagogik, zur Sozialpädagogik, zur Geschichte der Pädagogik, Ästhetik und Bildungstheorie zeigen sein kulturtheoretisch akzentuiertes Interesse an der Klärung der Fragen nach den Konstitutionsbedingungen von Subjektivität als Frage von Bildungsprozessen. Die zentrale Aufgabe der Sozialpädagogik sieht er in den Prozessen der Bildung und Erziehung. Durch Mündigkeit soll eine Erziehung zur Selbstverantwortung und Spontanität erreicht werden (Mollenhauer 1968).

Vermittelt durch sein Spätwerk, die Arbeiten zur sozialpädagogischen Diagnostik, befasst er sich (in Kooperation mit Uhlendorf) mit dem Verhältnis von Individuum und Gesellschaft in beschädigten Lebenslagen und verknüpft damit - je nach Etappe schwächer oder stärker - die Frage nach dem Pädagogischen durch eine gesellschaftstheoretische Reflexion der Bedingungen und Möglichkeiten von Pädagogik.

Michael Winkler (1995) hingegen entwirft eine Theorie der Sozialpädagogik, die von einem primär disziplinär geprägten Begriff der Sozialpädagogik ausgeht, der am Diskurs der Theoriekonstitution orientiert ist (1995). Sozialpädagogik orientiert sich an den Erkenntnissen der Praxis, sie bezieht sich auf die soziale Praxis und steht mit ihr in einem reflexiven Zusammenhang. Theorie und Praxis, Empirie und Reflexion, Denk- und Handlungsmuster werden immer wieder einer kritischen Prüfung unterzogen, um neue Sicht- und Interpretationsweisen auf Problemlagen von Einzelnen und Gesellschaft zu entwickeln. Theoriebegriffe können in Winklers Verständnis nicht starr sein, sondern müssen Kategorien sein, die durch Prinzipien gefüllt werden und gleichzeitig zeitdiagnostische Substanz aufweisen. Für Michael Winkler ist die Beobachtung, Analyse und Begründung des empirischen Diskurses wesentlich, da durch die Reflexion des Diskurses die kommunikativen Strukturen in der Sozialpädagogik zum sozialpädagogischen Handeln werden. Theorie der Sozialpädagogik schließt zwei Ebenen mit ein, die reale Gegenstandstheorie, die Beschreibung, Analyse der Voraussetzungen, Bedingungen, Strukturen, Funktionen und Grenzen und reflexiven Durcharbeitung dieser Elemente in Sinn einer sozialen Konstruktion. Die Inklusionsarbeit der Sozialpädagogik besteht in ihrem Beitrag zur gesellschaftlichen Integration. Probleme und Risiken werden sozialpädagogisch bearbeitet, die unter Mitwirkung der Akteure und Institutionen in der Sozialen Arbeit in der Gesellschaft produziert werden. Damit trägt die Soziale Arbeit zur Herstellung der sozialen Infrastruktur bei. Sozialpädagogik im Sinne Winklers interessiert sich für die Kompetenzen, Bildungs- und Entwicklungsprozesse einzelner Akteure, um Lebensbedingungen zu schaffen, die dauerhafte Existenzen jenseits der Armut sichern. Der Aufklärungsimpetus, aber auch das kritische Moment in den Arbeiten von Michael Winkler liegt in der Stärkung des Subjektbegriffs als eigenständiges Konstrukt in der Sozialen 
Arbeit, der dazu dient zu fragen, ob sozialpädagogische Institutionen und Orte die Subjektivität einschränken oder schädigen und welche individuellen Möglichkeiten die Akteure Sozialer Arbeit haben, um ein selbstbestimmtes Leben zu führen (1995). In ähnlicher Weise wurde dieser Ansatz, den Akteur der Sozialen Arbeit in den Mittelpunkt des theoretischen Interesses zu stellen schon von der Bildungstheorie aufgegriffen, jedoch mit deutlicher Fokussierung auf die Herstellung von Bildungsprozessen.

Das Konzept der Alltags- und Lebensweltorientierung von Hans Thiersch (2008) und das zu Sozialstaat, Pädagogik und Lebensbewältigung von Lothar Böhnisch (1988) ${ }^{15}$ wurde als Kritik an und Gegenorientierung zu einer zunehmenden Institutionalisierung, Spezialisierung und Professionalisierung im Spannungsfeld modernisierungstheoretischer Diskurse entwickelt. Ziel war es eine verstärkte Hinwendung der Sozialen Arbeit zu den Adressaten zu erreichen. Damit schließt insbesondere Hans Thiersch an alte Traditionslinien, wie z. B. von Alice Salomon an, die mit ihren Konzepten die Professionalisierung und Institutionalisierung von Sozialer Arbeit erst ermöglicht haben. Ziel war es, durch Reformen alle sozialen Bereiche neu zu gestalten. Hans Thiersch und Lothar Böhnisch fordern ein verändertes Selbstverständnis der Sozialen Arbeit ein; es geht um eine Erweiterung der Funktionsbestimmung und des Gegenstandes Sozialer Arbeit, um Strategien der Lebensbewältigung, um in deren Folge einen gelingenden Alltag für die Adressatinnen zu ermöglichen. Dabei ist eine spezifische Methodisierung von Verstehen und Handeln in der Sozialpädagogik zentral. Sie eröffnete eine neue Perspektivierung auf Praxis durch die Vermittlung von sozialwissenschaftlichen Erkenntnissen, um eine kritische Analyse und Aufklärung der gesellschaftlichen und insbesondere der politischen Verhältnisse zu ermöglichen.

Im Anschluss an die Tradition des Westlichen Marxismus - mit besonderem Bezug auf die von Lefebvre vorgestellte materialistische Alltagstheorie sowie die kritische Theorie der Bildung Heydorns - ist Heinz Sünker an einer gesellschaftsanalytischen Grundlegung Sozialer Arbeit interessiert, die als emanzipatorisch zu verstehen ist, wenn sie an kapitalistischen Verhältnissen im Sinne einer Demokratisierungsperspektive zu arbeiten beabsichtigt (Sünker 1989)16. Dies stellt eine besondere Herausforderung unter den Bedingungen einer kapitalistischen Gesellschaftsformation dar, in der die Klassenstruktur auf der Reproduktion sozialer Ungleichheit aufruht und Macht und Herrschaft den historisch erreichten Möglichkeiten von Gerechtigkeit und Gleichheit widersprechen. Soziale Arbeit, die an einer

\footnotetext{
${ }^{15}$ Dabei hat Böhnisch mit seiner Studie „Der Sozialstaat und seine Pädagogik“ (1988) in den achtziger Jahren des letzten Jahrhunderts eine der drei grundlagentheoretisch ausgerichteten Habilitationsschriften vorgelegt, die bis heute für die Theoriebildung relevant sind - Winkler mit „Eine Theorie der Sozialpädagogik“ (1988) und Sünker mit „Bildung Alltag und Subjektivität. Elemente zu einer Theorie der Sozialpädagogik“ (1989) sind die beiden anderen Autoren.

16 Analytisch werden diese Leitmotive, die mit der Frage nach der Konstitution von Sozialetat und Demokratisierung im Zentrum finden, auch für Hornstein (1995) und Schaarschuch (1995) bedeutsam.
} 
Überwindung von Routine und dem Kurieren an Symptomen in unterschiedlichen Praxisfeldern interessiert ist, muss sich unter den gegenwärtigen Bedingungen der Frage nach Begründungen für Arbeitsansätze erneut und erneuert stellen. Das macht die Frage nach der politischen Produktivität sozialer Arbeit virulent und zeigt die Bedeutung der Frage nach den Konstitutionsbedingungen von Subjektivität als Frage nach Reflexivität, gesellschaftliche Urteilskraft und politischer Handlungskompetenz - dies auf Seiten von Professionellen und Klienten Sozialer Arbeit.

Während Heydorns Analyse den Vermittlungen zwischen Bildung und Gesellschaftsgeschichte sowie Bildung und Politik gilt, dabei ihr Zentrum in den Bildungsprozessen von Individuen und deren Bewusstseins - wie Handlungsformen hat, geht Lefebvre davon aus, die Kritik des Alltagslebens schließe die Kritik der politischen Ökonomie im Sinne von Marx ein und übergreife diese, sei darauf ausgerichtet, den gesellschaftlichen Menschen, der auf seinen ökonomischen Aktivitäten, aber eben auch darüber hinaus beruhe, zu erforschen (Lefebvre 1989: 604)17.

Gegen jeden Ökonomismus hält dabei eine kritische Theorie der Gesellschaft daran fest, dass im Alltagsleben die Menschen sich ihrer Subjektivität und damit ihrer Handlungsfähigkeit bewusst werden müssen bzw. darin ihre Subjektivität zu entwickeln haben. In diesem Rahmen ist bedeutsam, dass materialistische Alltagstheorien, die den Anspruch einer erkenntniskritischen Darstellung der Vermittlung von Makro-, Meso- und Mikroprozessen im Bereich der Konstitution gesellschaftlicher Formen und deren Auswirkungen auf die Verfasstheit individueller Existenz erheben, zu einer Präzisierung von Problemstellungen wie zu einer Perspektivenerweiterung sowohl in Bezug auf die Vergesellschaftungsfrage als auf die Bildungsfrage führen.

So argumentiert Lefebvre um der Subjektperspektive willen für eine kulturrevolutionär begründete Änderung der gegenwärtigen Gesellschaft, damit für eine Auflösung der verdinglichten und verdinglichenden Strukturen des Alltagslebens. Alltagstheorie und Bildungstheorie vermittelnd und den Anschluss an die philosophische Tradition herstellend, dabei die sokratische Mäeutik als Fokus benutzend, beinhaltet dies für Lefebvre, „der Alltäglichkeit zu helfen, eine in ihr anwesendeAbwesende Fülle zu erzeugen“ (Lefebvre 1972: 31). Mit dieser Positionierung ist zugleich der Ausgangspunkt für seine Analysen benannt, da er sich durchgängig

\footnotetext{
17 Damit ergibt sich eine Vermittlungsmöglichkeit zu Adornos Analyse des Verhältnisses von Gesellschaft und Individuum, die für Soziale Arbeit grundlegend ist: Zunächst hält Adorno fest, „dass der Begriff der Gesellschaft eigentlich ein Begriff ist, der ein Verhältnis zwischen Menschen bezeichnet“ (Adorno ([1968] 1993): 68). Dann führt er aus: „Es gibt also genauso wenig im gesellschaftlichen Sinn Individuen, nämlich Menschen, die als Personen mit eigenem Anspruch und vor allem als Arbeit Verrichtende existieren können und existierten, es sei denn mit Rücksicht auf die Gesellschaft, in der sie leben und die sie bis ins Innerste hinein formt, wie es auf der anderen Seite auch nicht Gesellschaft gibt, ohne dass ihr eigener Begriff vermittelt wäre durch die Individuen; denn der Prozess, durch den sie sich erhält, ist ja schließlich der Lebensprozess, der Arbeitsprozess, der Produktion -und Reproduktionsprozess, der durch die Einzelnen, in der Gesellschaft vergesellschafteten Individuen in Gang gehalten wird“ (ibidem: 69-70).
} 
von der Einsicht in die Notwendigkeit einer Rehabilitierung des Alltagslebens leiten lässt (Lefebvre 1977: I, 134-135). Die Forderung nach einer Umwandlung des Alltagslebens lebt vom Aufweis des verborgenen Reichtums der Welt der Trivialitäten und führt zu der Feststellung, das Alltagsleben stehe in wesentlicher Beziehung zu allen Tätigkeiten und umfasse sie mitsamt ihren Konflikten und Differenzen. Liegt mithin im täglichen Leben der „rationelle Kern, das wirkliche Zentrum der Praxis“ (Lefebvre 1972: 49), findet die Produktion und Reproduktion gesellschaftlicher Beziehungen im Alltagsleben ihre Grundlegung, dann wird auch die elementare Bedeutung des Nachweises vom geschichtlichen Charakter des Alltagslebens einsichtig. Lefebvre fundiert diesen historischen Charakter durch eine Rekonstruktion und Aufschlüsselung der historisch-strukturellen Genese differierender Ausformungen des Alltagslebens im Zusammenhang gesellschaftlich, also praktisch, vermittelter Bedingungen.

Lefebvres Vorgehensweise, die seine Theorieentwicklung an die Entwicklung des Gegenstandes, des Alltagslebens also, bindet, führt zur Einsicht in den mehrdeutigen und mehrwertigen Charakter des Alltagslebens, was er mit immer neuen Bestimmungen und Annäherungen, den changierenden Gestaltungen seines Gegenstandes entsprechend, zu präzisieren und einzufangen sucht. Der kategoriale Oberbegriff für die Beschreibung und Analyse des Alltagslebens ist der der „Ambiguität" (Lefebvre 1975: 14).

Verstärkt wird die Bedeutung der Ambiguität durch die Entwicklung vom Alltagsleben zur Alltäglichkeit, die diese als „bürokratische Gesellschaft des gelenkten Konsums“ bestimmt (Lefebvre 1972: 99-154). Die Konsolidierung dieser Alltäglichkeit als die defiziente Form von Alltagsleben ist ein Charakteristikum spätkapitalistischer Gesellschaften, was der Unterstützung durch eine dreifache Bewegung bedarf: erstens bedarf es einer Vergesellschaftung in der Form einer „Totalisierung der Gesellschaft“; zweitens auf der Seite von Subjektentwicklung der Form einer „extremen Individualisierung“ sowie drittens einer „Partikularisierung“ (Lefebvre 1978: 140). Praktisch bedeutend wird dies für Soziale Arbeit, weil das Netz der Gesellschaft immer enger, die individuelle Existenz aber immer atomistischer wird $^{18}$. Gesellschaftspolitisch wie gesellschaftstheoretisch sowie mit Bezug auf die Frage nach Strukturen wie Inhalten von Bildungsprozessen entscheidend ist dabei, dass diese Etablierung der "Alltäglichkeit als verallgemeinerter Lebensweise“ Lefebvre 1975: 225) mit einem Prozess der inneren Kolonisierung einhergeht (Lefebvre 1972: 86; 1975: 242), der als Zugriff auf Körper und Sinne, Raum und Zeit wie in Prozessen der Parzellierung, der Zerschlagung von Lebenszusammenhängen stattfindet. Diese Lebensweise ist damit insgesamt durch eine Tendenz zu Passivität und Nicht-Partizipation gekennzeichnet (Lefebvre 1975: 120-121).

\footnotetext{
18 Strukturell gibt es damit eine enge Verknüpfung zwischen den Ergebnissen der Gesellschaftsanalyse Lefebvres mit der Adornos.
} 
Vor dem Hintergrund dieser Gefährdung des Menschen und der Welt stellt sich die Frage nach Alternativen. Es geht um ein Erkennen der Alltäglichkeit wie deren Veränderbarkeit, so dass Rekonstruktionsarbeit als handelndes Erkennen (ibidem: 122) ein Erfassen der Alltäglichkeit ist, das dem Leben in der Alltäglichkeit wie der kritischen Distanz zu dieser Alltäglichkeit entspricht (Lefebvre 1972: 105) und selbst die Basis für die Bildung von Widerständigem verkörpert. Wesentlich ist dabei allerdings die neue qualitative Dimension, die in Folgendem liegt: „Der Akt, der Erkenntnis und Praxis inauguriert, ist poietisch“ (Lefebvre 1975: 123). Poiesis als erkennendes Handeln und als schöpferisches Erkennen verweist auf Dimensionen eines kreativen Vermögens, das auf die - auch ästhetisch zu fundierende Bedeutung von „Spontaneität“ als „neue, wiedergewonnene Spontanität“ oder -so Heydorns (1979) bildungstheoretische Lesart als „rational vermittelte Spontanität“ (1979) - zu beziehen ist.

Da es um ein gesellschafts- wie kulturanalytisch begründetes „revolutionäres Projekt einer Befreiung“ (Lefebvre 1972: 24) und damit um eine „Transformation des Alltagslebens" geht (Lefebvre 1978: 44, 231), bedarf dies einer bildungspolitischen wie bildungspraktischen Unterfütterung. Denn eine kritische Bildungstheorie ist als Theorie der Befreiung des Menschen zu verstehen (Heydorn 1979; vgl. Sünker 2006b), so dass Soziale Arbeit in diesem Ansatz als Bildungsarbeit verstanden wird.

Ein kritisches Bildungskonzept thematisiert dabei das spannungsvolle Verhältnis von Individuum und Gesellschaft seit seinen Anfängen in der Antike. Die von Heydorn herausgearbeiteten Konstitutionsbedingungen von Subjektivität im Kontext der Dialektik von institutionalisierter Bildung und menschlicher Befreiung ist für Konzepte der Sozialen Arbeit zu nutzen. Sozialpädagogik und Bildungstheorie verhalten sich daher nicht nur in ihren Orientierungen komplementär zueinander. Die Theorie der Bildung ist fundierend, wenn Sozialpädagogik nicht nur Normalisierungsarbeit sein soll, sondern auch der Initiierung und Beförderung von Bildungsprozessen dient. Das Verständnis von Sozialpädagogik als einer Form institutionalisierter Bildung - und ihres Widerspruchs - kann ein Beitrag zur Bearbeitung des dringlichen Problems der Konstitution und Reproduktion sozialer Ungleichheit sein (Sünker 1995a).

Sozialpädagogik ist aufgrund ihrer gesellschaftlichen-politischen Einbettung nicht abgeschlossen, sondern muss infolge ihrer Konstitutionsbedingungen immer wieder neu aufgeschlossen und in ihren analytischen wie praktischen Konsequenzen reflektiert werden ${ }^{19}$.

\footnotetext{
${ }^{19} \mathrm{Vgl}$. Anmerkung 1 (!).
} 


\section{Literaturverzeichnis}

Adorno Th. W. ([1968] 1993) Einleitung in die Soziologie, Frankfurt/M., Suhrkamp.

Böhnisch L. (1988) Der Sozialstaat und seine Pädagogik, Neuwied, Luchterhand.

Bühler-Niederberger D., Sünker H. (2014) Die proletarische Kindheit in: M. S. Baader, F. Eßer, W. Schröer (Hg.), Kindheiten in der Moderne, Frankfurt/M., Campus: 72-96.

Füssenhäuser C. (2005) Werk (Geschichten) der Sozialpädagogik, Baltmannsweiler, Schneider Hohengehren.

Geremek B. (1988) Geschichte der Armut, München, Artemis.

Hartwich H. (1977) Sozialstaatspostulat und gesellschaftlicher Status quo, 2nd ed., Opladen, Westdeutscher Verlag.

Hegel G. W. F. (1955) Grundlinien der Philosophie des Rechts, Hamburg, Meiner.

Heydorn H.-J. (1979) Über den Widerspruch von Bildung und Herrschaft, Frankfurt/M., Syndikat.

Hornstein W. (1995) Zur disziplinären Identität der Sozialpädagogik in: H. Sünker (Hg.) (1995) Theorie, Politik und Praxis Sozialer Arbeit, Bielefeld, Kleine: 12-33.

Lenhardt G., Offe C. (1977) Staatstheorie und Sozialpolitik in: C. v. Ferber, Kaufmann (eds.): 98-127.

Kocka J. (1990a) Weder Stand noch Klasse. Unterschichten um 1800, Bonn, Dietz.

Lefebvre H. (1972) Das Alltagsleben in der modernen Welt, Frankfurt/M., Suhrkamp.

Lefebvre H. (1975) Metaphilosophie, Frankfurt/M., Suhrkamp.

Lefebvre H. (1977) Kritik des Alltagslebens, Kronenberg, Athenäum.

Lefebvre H. (1978) Einführung in die Modernität, Frankfurt/M., Suhrkamp.

Lefebvre H. (1989) La somme et le reste, Paris, Meridien Klincksiek.

Marx K. o. J.: Grundrisse der Kritik der politischen Ökonomie (Rohentwurf) 1857-1858, Frankfurt, EVA.

Mollat M. (1987) Die Armen im Mittelalter, München, Beck. 
Mollenhauer K. (1959) Die Ursprünge der Sozialpädagogik in der industriellen Gesellschaft. Eine Untersuchung zur Struktur pädagogischen Denkens und Handelns, Weinheim und Berlin, Belz.

Mollenhauer K. (1968) Erziehung und Emanzipation: polemische Skizzen, München, Juventa.

Ortmeyer B. (2009) Mythos und Pathos statt Logos und Pathos. Zu den Publikationen führender Erziehungswissenschaftler in der NS-Zeit: Eduard Spranger, Hermann Nohl, Erich Weniger und Peter Petersen, Weinheim/Basel, Beltz.

Otto H.-U. (1973) Professionalisierung und gesellschaftliche Neuorientierung. Zur Transformation des beruflichen Handelns in der Sozialarbeit in: H.-U. Otto, S. Schneider (eds.) Gesellschaftliche Perspektiven der Sozialarbeit, Bd. 1. Neuwied, Luchterhand.

Otto H.-U., Thiersch H. (eds.) (2014) Handbuch Soziale Arbeit, 4. Aufl., München, Reinhardt.

Peukert D. (1986) Grenzen der Sozialdisziplinierung. Aufstieg und Krise der deutschen Jugendfürsorge von 1878 bis 1932, Köln, Bund.

Peukert D. (1989) Sozialpädagogik in: D. Langewiesche, H.-E. Tenorth (eds.), Handbuch der deutschen Bildungsgeschichte. Band V 1918-1945. Die Weimarer Republik und die nationalsozialistische Diktatur, München, Beck: 307-336.

Raphael L. (2004) Königsschutz, Armenordnung und Ausweisung - Typen der Herrschaft und Modi der Inklusion und Exklusion von Armen und Fremden im mediterraneuropäischen Raum seit der Antike in: A. Gestrich, R. Lutz (Hg.), Inklusion/Exklusion. Studien zu Fremdheit und Armut von der Antike bis zur Gegenwart, Frankfurt a.M. u.a., Peter Lang: 15-34.

Richter E. (2004) Jugendarbeitslosigkeit und Identitätsbildung. Sozialpädagogik zwischen Arbeitserziehung und Vereinspädagogik: Eine historisch-systematische Rekonstruktion. Frankfurt a.M. u.a., Peter Lang.

Sachße Ch., Tennstedt F. (1980) Geschichte der Armenfürsorge in Deutschland. Vom Spätmittelalter bis zum 1. Weltkrieg, Stuttgart, Kohlhammer.

Sachße Ch., Tennstedt F. (1986) Soziale Sicherheit und soziale Disziplinierung. Beiträge zu einer historischen Theorie der Sozialpolitik, Frankfurt, Suhrkamp.

Sachße Ch., Tennstedt F. (1995) Historische Forschung zur Sozialarbeit/Sozialpädagogik. Eine Zwischenbilanz nach 20 Jahren in: H. Thiersch, K. Grunwald (eds.), Zeitdiagnose Soziale Arbeit. Weinheim, Juventa. 
Salomon A. ([1901] 1997) Frauenemanzipation und soziale Verantwortung. Ausgewählte Schriften Bd. 1: 1896-1908, München, Luchterhand.

Salomon A. (1913) Geschichte der sozialen Frauenarbeit in: Volkswirtschaftliche Blätter, zugleich Mitteilungen des Deutschen Volkswirtschaftlichen Verbandes, 12. Jg., Nr. 24, 31. Dez. 1913, S. 322f.

Salomon A. ([1918] 2000) Frauenemanzipation und soziale Verantwortung. Ausgewählte Schriften Bd. 2: 1908-1918, München, Luchterhand.

Salomon A. ([1921] 2004) Die sittlichen Grundlagen und Ziele der Wohlfahrtspflege in: Salomon A. ([1921] 2004) Frauenemanzipation und soziale Verantwortung. Ausgewählte Schriften Bd. 3: 1918-1948, München, Luchterhand.

Schaarschuch A. (1995) Das demokratische Potential Sozialer Arbeit in: H. Sünker (Hg.) (1995) Theorie, Politik und Praxis Sozialer Arbeit, Bielefeld, Kleine: 48-71.

Schnurr S. (1988) Vom Wohlfahrtsstaat zum Erziehungsstaat. Sozialpolitik und soziale Arbeit in der Weimarer Republik und im Nationalsozialismus in: Widersprüche, Jg. 8, Heft 26: 47-64.

Schnurr S. (1997) Sozialpädagogen im Nationalsozialismus. Eine Fallstudie zur sozialpädagogischen Bewegung im Übergang zum NS-Staat. Weinheim und München, Juventa.

Steinacker S. (2007) Der Staat als Erzieher. Jugendpolitik und Jugendfürsorge im Rheinland vom Kaiserreich bis Zum Ende des Nazismus. Stuttgart, Verlag.

Steinacker S., Sünker, H. (2010) Politische Kultur, Demokratie und Bildungspraxis in Deutschland. Mitverwaltung - Selbstbestimmung - Partizipation oder "1968“ im Kontext von Geschichte, „Zeitschrift für Pädagogik“ 56 (No. 1): 22-36.

Steinmetz G. (1993) Regulating the Social. The Welfare State and Local Politics in Imperial Germany, Princeton, Princeton University Press.

Sünker H. (1989) Bildung, Alltag und Subjektivität. Elemente zu einer Theorie der Sozialpädagogik, Weinheim, Deutscher Studien Verlag.

Sünker H. (1991) Nationalsozialistische Herrschaftssicherung durch Soziale Arbeit: Destruktion wohlfahrtsstaatlicher Ansätze und hilfepolitischer Diskurse in der „Volkspflege“ in: Ch. Berg, S. Ellger-Rüttgarth, „Du bist nichts, Dein Volk ist alles“. Forschungen zum Verhältnis von Pädagogik und Nationalsozialismus, Weinheim, Deutscher Studienverlag: 46-59.

Sünker H. (Hg.) (1995) Theorie, Politik und Praxis Sozialer Arbeit, Bielefeld, Kleine. 
Sünker H. (1995a) Soziale Arbeit und Vergesellschaftung der Gesellschaft in: H. Thiersch, K. Grunwald (eds.) (1995) Zeitdiagnose Soziale Arbeit, Weinheim, Juventa: 185-210.

Sünker H. (Hg.) (1995b) Theoretische Ansätze, gesellschaftspolitische Kontexte und professionelle Perspektiven Sozialer Arbeit in: H. Sünker (Hg.) (1995) Theorie, Politik und Praxis Sozialer Arbeit, Bielefeld, Kleine: 72-101.

Sünker H. (2003) The Politics of Social Welfare: Critical Perspectives on Contemporary Social work in: H. Sünker, R. Farnen, G. Széll (eds.) Political Socialisation, Participation and Education, Frankfurt/M., Peter Lang: 113-130.

Sünker H. (2006a) Community's Discontent: the ideology of Volk community in National Socialism, "Policy Futures in Education" 4 (no. 3): 306-319.

Sünker H. (2006b) Politics, Bildung and Social Justice. Perspectives for a Democratic Society, Rotterdam/Taipei, Sense.

Thiersch H., Grunwald K. (eds.) (2008) Praxis der Lebensweltorientierten Sozialen Arbeit, Weinheim, Juventa.

Thiersch H., Grunwald K. (eds.) (1995) Zeitdiagnose Soziale Arbeit. Weinheim, Juventa.

Thole W. (2005) Grundriss Soziale Arbeit, Wiesbaden, VS Verlag.

Vester M. et al. (2001) Soziale Milieus im gesellschaftlichen Strukturwandel. Zwischen Integration und Ausgrenzung, Frankfurt/M., Suhrkamp.

Wehler H.-U. (1987) Deutsche Gesellschaftsgeschichte 1700 - 1815, München, Beck.

Winkler M. (1988) Eine Theorie der Sozialpädagogik, Stuttgart, Klett-Cotta.

Winkler M. (1995) Die Gesellschaft der Moderne und ihre Sozialpädagogik in: H. Thiersch, K. Grunwald (eds.), Zeitdiagnose Soziale Arbeit, Weinheim, Juventa: 155-184.

Rita Braches-Chyrek, Heinz Sünker

\section{Pedagogika społeczna/praca socjalna w Niemczech}

Wraz z dysertacją Klausa Mollenhauera Die Ursprünge der Sozialpädagogik in der industriellen Gesellschaft (1959) [Początki pedagogiki społecznej w przemysłowym społeczeństwie] w postfaszystowskiej Republice Federalnej Nie- 
miec rozpoczęła się pierwsza krytyczna dyskusja nad warunkami konstytuowania się konceptu pedagogiki społecznej adekwatnego do przedmiotu formułowanego $\mathrm{w}$ teorii i w praktyce. Oznaczało to przezwyciężenie dominującej wówczas pedagogiki humanistycznej (Geisteswissenschaftlichen Pädagogik) - konceptualnie ograniczającej się do stosunku wychowawcawychowanek - co wyrażało się w szukaniu takiego rozumienia nauki o wychowaniu, która nie jest 'społecznie ślepa', która dopuszcza pedagogikę społeczną jako szczególną problematyzację społecznego wymiaru kształtowania się człowieka.

Przedłożony tekst - przyjmujący powyższe sformułowanie problemu - rekonstruuje historyczne i systematyczne warunki rozwoju pedagogiki społecznej/pracy socjalnej na ziemiach niemieckich. Analiza koncentruje się wokół tezy, iż pedagogika społeczna, stając wobec kapitalistycznego wzoru uspołecznienia, towarzyszącego mieszczańsko-kapitalistycznej formacji społecznej, musi w szczególny sposób zajmować się kształtowaniem stosunków między społeczeństwem/strukturami społecznymi a jednostką, a tym samym warunkami konstytucji podmiotowości, zdolnością do działania itd.

Na wstępie warto zauważyć, iż zarówno w ujęciu historycznym, jak i systematycznym, w okresie przedkapitalistycznym (przede wszystkim w średniowieczu) jak i kapitalistycznym, w różnych formach społeczności i społeczeństwa, chodzi zawsze o społeczną integrację ludzi. Prowadząc analizę społeczną, można odczytać funkcję pedagogiki społecznej jako uczestniczenie w rozgrywce między pomocą i kontrolą prowadzonymi przez państwo.

Rekonstruując historię pedagogiki społecznej/pracy socjalnej jako dyscypliny i jako profesji, należy zauważyć, iż jej początki w krajach niemieckich, podobnie jak w Europie, łączy się z instytucjonalizacją pomocy ubogim (początek XVI w.). Sprzeczności tkwiące w mieszczańsko-kapitalistycznej formacji wymagają sprawowania kontroli nad członkami społeczeństwa, chodzi bowiem o zabezpieczenie produkcji towaru, jakim jest 'siła robocza'. Ta kontrola może być ewentualnie wzmacniana środkami pedagogicznymi.

Przyglądając się etapom historycznego rozwoju Niemiec - Cesarstwo, Republika Weimarska, narodowy socjalizm, Niemiecka Republika Federalna - wyraźnie widać zróżnicowanie społeczno- i socjalno-politycznych podstaw teorii i praktyki społeczno-pedagogicznej. Aktualnym jest pytanie o warunki pedagogiki społecznej/pracy socjalnej w społeczeństwie uznającym swoje państwo za socjalne. Ten socjalny wymiar państwa rozpatrywany jest z dwóch perspektyw: ochrony lojalności (obywateli) i demokratycznego rozumienia społeczeństwa. 
Pedagogika społeczna/praca socjalna staje wobec różnych możliwości ujmowania sprzeczności między pomocą a kontrolą - między wzmocnieniem dyscyplinowania a wzmocnieniem emancypowania. Nie można przy tym zapomnieć, że w ostatnich 15 latach ukierunkowanie na emancypację podlegało coraz silniejszym neoliberalnym imperatywom, tak że w konsekwencji można wręcz mówić o powrocie do 'starej' logiki pomocy ubogim. Dlatego też powraca się do analizowanego już (przede wszystkim w latach 19681975) pytania o możliwości takiej pedagogiki społecznej/pracy socjalnej, która jest krytyczna wobec społeczeństwa, i do pytania o jej polityczną produktywność.

Tłumaczenie: Danuta Urbaniak-Zając 